\title{
Reduction of Visual Disruptions in Coronary Cine- Angiography for Better Diagnostic Viewing
}

\author{
K.A.S.H.Kulathilake, W.S.Wajiramali, D.W.J.Chathurika, D.A.N.Gunasekara, I.A.M.S.Ranasinghe, R.R.C.P.Rathnayake
}

\begin{abstract}
Coronary angiography is an invasive medical imaging modality which is widely used as a preliminary diagnostic method in Interventional Cardiology for detection of luminal obstructions in Coronary Arteries (CA). Noise, nonuniform illumination, low contrast and epicardial motion are considered as the reported visual degradations which are automatically recorded in Coronary angiography. Further, those factors form certain unpredictable obstructions during the angiography based disease diagnosis. In this study, it has been proposed a frequency filtering based frame enhancement method for surmounting those problems and improving the visual quality of the angiograms. The proposed method is based on homomorphic butter worth high pass filter and empirically validated contrast stretching technique. Further, the optical flow based frame stabilization method has been applied in the later stages of the proposed method for correcting the epicardial deformations. The results of the study clearly emphasized the visual improvement obtained from this method. The enhanced angiography frames produced in this study can be recommended for reliable stenosis detection. Further, these enhanced frames can be further used for quantitative stenosis assessment.
\end{abstract}

Keywords - Homomorphic filtering, optical flow, angiogram enhancement, non-uniform illumination, noise, CLAHE.

\section{INTRODUCTION}

$\mathrm{C}$ oronary angiography is a gold standard invasive medical image modality which is widely used in Interventional Cardiology to detect luminal obstructions in Coronary Arteries (CA). During the angiography procedure, a contrast dye will be injected into the CA through a catheter and blood flow through CA is recorded using an X-ray system called fluoroscopy. Angiogram can be produced either as still images or video and the video based coronary angiogram is known as Coronary Cine-angiogram (CCA).

Even though the angiography is used as one of the mandatory diagnosis modalities in Percutaneous Coronary Intervention (PCI), it has been reported that the physical organization of the organs in the chest and the artefacts that occur during the angiogram procedure make visual degradations in the recorded CCAs. Moreover, these factors formulate some visual disturbances in subjective stenosis analysis. In addition to that, the recent research attempts have

Manuscript received on $15^{\text {th }}$ Dec, 2017. Recommended by Dr. M. G. N. A. S. Fernando on $12^{\text {th }}$ June, 2018.

This paper is an extended version of the paper "Improving Diagnostic Viewing of Coronary Cine-Angiogrphy through Frequency Filtering based Frame Enhancement Method" presented at the ICTer 2017.

K.A.S.H. Kulathilake is a Senior Lecturer at the Department of Physical Sciences, Rajarata University of Sri Lanka. (shkulathilake@yahoo.com). W.S.Wajiramali, D.W.J.Chathurika, D.A.N.Gunasekara, I.A.M.S.Ranasinghe and R.R.C.P.Rathnayake are from Department of Physical Sicences, Rajarata University of Sri Lanka.

(shashiwarna92@gmail.com, jayanichathurikajd@gmail.com, achininalika6@gmail.com,madusha92@yahoo.com,

chnadee23@gmail.com). reported that these visual degradation factors cause some hindrances when formulating objective stenosis diagnosis approaches based on coronary angiography [1].

Overlapping vessels and superimposition of other organs such as ribs, spine or cardiac chambers on CA can be considered as degradation which occurs due to the physical organization of the organs in the chest [2][3]. Overlapped vessels make obstructions in vessel delineation. A clear recognition of Coromixing

nary Artery vasculature in angiography view is essential to segment the CA and locate the stenosis area [4]. This process becomes tedious or even impossible when the blood vessels in the image are overlapped. Superimposition of ribs, spine or cardiac chambers on blood vessels also provides unclear visualizations of $\mathrm{CA}$ in the angiogram image and causes obstructions to recognize the blood vessels clearly.

Non-uniform illumination, poor contrast, noise and motion are reported as the visual degradation factors that occur during the angiogram procedure [5][6][7]. Non-uniform illumination in CCAs visualizes an individual vessel breaking into several segments. Moreover, it makes some hindrances to clearly recognize which branch segment belongs to which Coronary Artery in the frame to be processed [1]. The poor contrast opacification of the vessel may lead to a false impression of an angiographically significant lesion or lucency, which could be considered a clot. Further, inadequate mixing of contrast material and blood could be seen as a luminal irregularity. It occurs when there is a strong presence of blood and contrast agents in the vessels, the thick vessels have more contrast to noise ratios than the small narrow ones [1][6]. Fig. 1(a) depicts the effect of nonuniform illumination. According to this figure, within the circular area, it is hard to identify which vessel branch belongs to which CA apparently. Moreover, Fig. 1(b) depicts the effect of a poor opacification. The contrast of the vessel depicted in the circular area is extremely poor when compared to the main blood vessel. In addition to the nonuniform illumination and poor opacification, CCA suffers from noise. The electrical system in cath-lab system adds Gaussian and impulse noise to the CCAs [8][9].
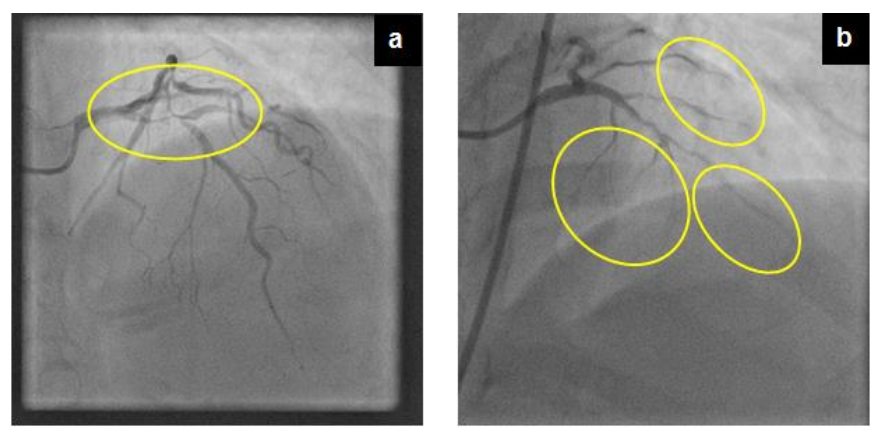

Fig. 1 Effect of non-uniform illumination and poor opacification of angiogram. (a) non-uniform illumination; (b) poor contrast. (The affected area is circled) 
In general, CCAs are recorded with a motion, which is another factor that affects the quality of the CCAs. Mainly, there are three types of motions in CCAs namely, global, radial and the local motions. Global and radial motions occur due to the systolic and diastolic movements of the heart [10]. Global motion produces rapid displacements of vessel structures from its' initial recorded point, and radial motion makes tiny movements in distal parts of the main CA. Local motion occurs due to the flow of the contrast agent within the CA. CA are adhering to the epicardium of the heart and follow dynamic performances of the related myocardium during heartbeat. Hence, the cardiac motion can be directly determined by arterial motion.

These visual hindrances create some obstructions when formulating an angiography based quantitative coronary analysis procedures. Nonetheless, the methodology suggested in this research study should be able to evade the aforementioned visual degradations in CCAs significantly. Even though the angiography has suffered from the visual degradations, it is still the most common image modality for clinicians to assess the severity of stenosis during the PCI [5]. One of the main reasons for that is, it provides excellent visualizations of arterial lumen, which can be used for stenosis diagnosis and treatment planning. Moreover, it is a low cost invasive image modality compared to IVUS, FFR and OCT techniques and the equipment is readily available in most hospitals, even in less-developed countries. In addition to that, all cardiologists are familiar with the equipment, and have experience in interpreting the resulting images. Further, the angiography is a relatively short imaging procedure, which facilitates effective clinical planning [11]. Hence, it has a great potential to improve the CCA to obtain an advanced visual quality. Moreover, it can be assisted to increase the level of accuracy of the medical judgments done using the CCAs.

In order to overcome the reported visual hindrances in CCAs, the frequency filtering based frame enhancement method has been implemented in this study. The proposed frame enhancement method is based on two major operations namely, homomorphic filtering and empirically validated contrast stretching technique. Moreover, the motion reduction of CCA is achieved through an optical flow based motion stabilization technique. The results of this study have proven the ability to obtain the uniformly illuminated noise reduced CCA frames. Moreover, the Left Main CA (LMA), Circumflex artery (CX) and Right CA (RCA) are considered as the main $\mathrm{CA}$ to be analysed under the selected angiography views.

The rest of the article is organized as follows: Next section elaborates the literature review of the study. Afterwards, the proposed frame enhancement method has been explained in detail. The experiment setup and the results are reported in the subsequent section. A discussion on the study results and the future work that can be extended from this study has been comprehensively elaborated in the final sections of this article.

\section{RELATED WORK}

Even though the coronary angiography consists of visual degradations, it is still the standard technique for guiding PCI in patients with CA disease [12]. Therefore, various research attempts have been made in recent past to improve the visualization of coronary angiography. Those published researches are based on various theories and techniques such as filtering, non-linear approach using stick, pseudo colour processing, contrast stretching and wavelet based noise removal. Moreover, there are some reported standards in the area of angiographic image enhancement, which have been widely accepted by cardiologists [13]. Those are; (i) the image enhancement is used for visualization purposes only, and not for quantitative analysis, (ii) detailed image structures should not be lost during the enhancing procedure and (iii) the original dimensions of vascular structures should be preserved in the enhanced image. Following sections elaborates the literature evidences under the aforementioned theories and techniques respectively.

It is apparent that various filtering techniques are being used for enhancing angiography images by reducing noise and non-uniform illumination. The angiogram enhancement technique mentioned in [14] is based on directional filter banks and those directional filters were implemented using high pass filters. The directional images obtained from the filter bank could be considered as the results obtained by the decomposition of the original image based on the gradient direction. Further, it has the capability of reducing the noise as an added advantage. An improved multi-scale Hessian matrix combined with morphological top-hat operation has been implemented in resent research study to enhance angiography [15]. The main objective of this study was to suppress non-vascular structure and improve the profile of small tiny blood vessels recorded in angiography.

The noise associated with the images is also increased during the traditional enhancement techniques, which are based on unsharp masking. Tu and his team have published a non-linear model for enhancing the angiography image details without having such negative effects and that suggested model was known as stick-guided lateral inhibition [6]. This proposed model simulates the enhancing mechanisms integrated in the eyes of human beings and of many animals and its implementation is based on asymmetric sticks [16]. The sticks are a set of line segments passing through the current pixel and are able to approximate the edges in images without co-relating with noise patches. Therefore, sticks can smoothen out noise patches without damaging the real edges of the angiogram [16]. Results of this study have provided high observer agreement value for stick based method rather than unsharp mask based enhancement method.

Pseudo colouring of angiogram images can be used as an enhancement technique to improve its visual quality. It is done by mapping each pixel value in a grayscale image into a colour according to a table or function [8]. Pseudo colour processing could accentuate blood vessels in angiography images.

Contrast Limited Adaptive Histogram Equalization (CLAHE) method has been applied in a recent research study for improving the contrast of the angiograms during its preprocessing phase[17]. In CLAHE, the histogram is cut at some threshold and then the equalization is applied. It is an adaptive technique because an image is enhanced by applying CLAHE on small data regions called tiles rather than the entire image. The resulting neighbouring tiles are then combined back faultlessly using bilinear interpolation [18]. The contrast in the homogeneous region can be limited so that the noise amplification can be avoided [19]. 
Research study conducted for extracting the CA tree in [20] has applied "temporal" wavelet transformation for noise removal and matched filters for blood vessel enhancement. In wavelet transformation, the input image is decomposed into sub regions by using low pass and high pass filters. Hence, the noise is automatically shifted into the specific decomposed images. This noise has been removed by thresholding the image.

Motion is another negative effect on CCAs and motion stabilization becomes extra pre-processing activity in CCA based processing. However, it has been reported that the motion of anatomy is quiet complex and cannot be accurately corrected by estimating models with low degree of freedom such as rigid or affine transformation [21]. The literature survey of this study reveals that the feature descriptor based method, image registration and optical flow based methods are among the major techniques for motion stabilization in CCAs and provided acceptable results for determining the cardiac dynamics [1] [9] [22][23].

A Scale Invariant Feature Transform (SIFT) based motion estimation and video stabilization technique have been described in a recent research study [9]. In this study, SIFT has been used to obtain the key points between two consecutive frames. The shortest distance between SIFT feature in two consecutive frames were calculated during the SIFT feature matching step of this study to determine the Global Motion Vector (GMV). The optical flow based method was proposed by Meunier and team to compute the regional epicardial deformation from coronary cine angiograms [22]. The motion of the CA was tracked using the proposed algorithms as a whole and quantify the two dimensional deformation of the epicardial surface locally. Arterial dynamics was also analysed by the Zheng and Weirong using an optical flow based technique and elastic registration [24]. The improved template matching technique has been implemented in [1] to obtain the visual alignment of the CCAs frames.

\section{METHODOLOGY}

The proposed method for frame enhancement in CCA is explained in this section. The direct CCA frames denoted as $f_{0}(x, y)$ are the input to the proposed method and $(x, y)$ denotes the coordinates of the image function $f_{0}$. This proposed method iteratively processes the frames of input direct CCA sequentially and uniformly illuminated noise reduced enhanced frames are produced as output.

The proposed method consists of five major implementation phases namely; noise removal, application of homomorphic filter, contrast stretching, calculating motion vector and creating stabilized frame. The following sections briefly describe the important steps of each and every implementation phase of the proposed method.

\section{A. Noise Removal}

According to the literature review, it has been revealed that the angiogram images are recorded with salt and pepper noise. In order to eliminate the salt and pepper noise, a median filter with kernel size $3 \times 3$ was applied to the input direct CCA frame to be processed [9]. The frame obtained after the noise reduction is denoted as $f_{l}(x, y)$ and it can be characterized by two components namely illumination $(i)$ and reflectance $(r)$ as shown in (1); $f_{1}((x, y)=i(x, y) \times r(x, y)$

\section{B. Application of Homomorphic Filter}

Subsequently, this noise-reduced frame is further processed to remove the effect of non-uniform illumination. In order to achieve that, homomorphic butterworth high pass filter was applied to attenuate the illumination component $(i)$ from the CCA frame [14]. Homomorphic filtering is a method in which the illumination and reflectance components can be filtered individually. It consists of several steps and those steps have been elaborated subsequently.

Initially, the CCA frame $f_{l}(x, y)$ is mapped into the natural logarithm domain to transform the frame as a sum of its illumination and reflectance components. After that, the Discrete Fourier Transform (DFT) is applied to the frame to transform it into the frequency domain. Equation (2) represents the transformation of $f_{l}(x, y)$ into DFT;

$p(u, v)=i(u, v)+r(u, v)$

where, $i(u, v)$ and $r(u, v)$ are the Fourier transforms of $\ln \{i(x, y)\}$ and $\ln \{r(x, y)\}$.

Moreover, $(u, v)$ represents coordinates of the frequency spectrum obtained by the DFT.

It has been reported that the illumination components of the CCA frame can be easily identified through the low frequency content in the frequency domain because the illumination is considered as a slowly varying pattern in a particular image [14]. Hence, the butterworth high pass filter was applied to emphasize the high frequency components of the frame to be processed. These high frequency components are responsible for saving the ridge structures in the frame and for attenuating the low frequency bands, which are responsible for illumination. In order to filter out the high frequency bands, a convolution was done between the DFT frame $(p(u, v))$ and the butterworth high pass filter $(b(u, v))$ as shown in (3);

$h(u, v)=p(u, v) * b(u, v)$

where $h(u, v)$ denotes the resulting filtered frame obtained finally.

Moreover $b(u, v)$ is obtained multiplying the $\log$ transformed frame $\left(f_{l}(x, y)\right)$ by the butterworth high pass filter function ( $h p f)$ given in (4);

$$
h p f=\frac{1}{1+\left\{D_{0}+D(u, v)\right\}^{2 n}}
$$

where, $D_{0}$ is the distance from origin to cutoff frequency in the DFT frequency spectrum, $D(u, v)$ is the radial distance from the origin and $n$ is the order. According to the empirical results, values of both $D_{0}$ and $n$ have been set as 10 and 2 respectively.

After the filtering, inverse DFT has been applied to transform the image into natural logarithm domain. Then, to get back the homomorphic filtered CCA frame to the spatial domain, the transformation of natural logarithm has been inversed, which is exponential. Equation (5) represents this operation. 


$$
f_{2}(x, y)=i_{0}(x, y) \times r_{0}(x, y)
$$

where, $f_{2}(x, y)$ denotes the homomorphic filtered image. $i_{0}(x, y)$ and $r_{0}(x, y)$ are the illumination and reflectance components of the homomorphic filtered image where $i_{0}(x, y) \neq i(x, y)$ and $r_{0}(x, y) \neq r(x, y)$.

\section{Contrast Stretching}

Moreover, this uniformly illuminated frame has been normalized by using (6) to obtain a better contrast among the vessel structures [14].

$$
f_{3}(x, y)=\left\{\begin{array}{l}
M_{d}+\sqrt{\frac{V_{d}\left(f_{2}(x, y)-M\right)^{2}}{V}} \text { if }\left(f_{2}(x, y)>M\right. \\
M_{d}-\sqrt{\frac{V_{d}\left(f_{2}(x, y)-M\right)^{2}}{V}} \text { otherwise }
\end{array}\right.
$$

where $M$ and $V$ denote the estimated mean and variance of input frame $\left(f_{2}(x, y)\right)$ and $M_{d}$ and $V_{d}$ are desired mean and variance values respectively. $f_{3}(x, y)$ is the output frame.

As mentioned in [14], $M_{d}$ was set as $M / 2$ and $V_{d}$ was set as $(V \times 4)$ to obtain better results. It is important to note that the radial distance and order parameters of butterworth high pass filter and $M_{d}, V_{d}$ parameters of normalization are adjustable according to CCA obtained under different machines. Fig. 2 clearly depicts a visual illustration of this proposed frame enhancement method.

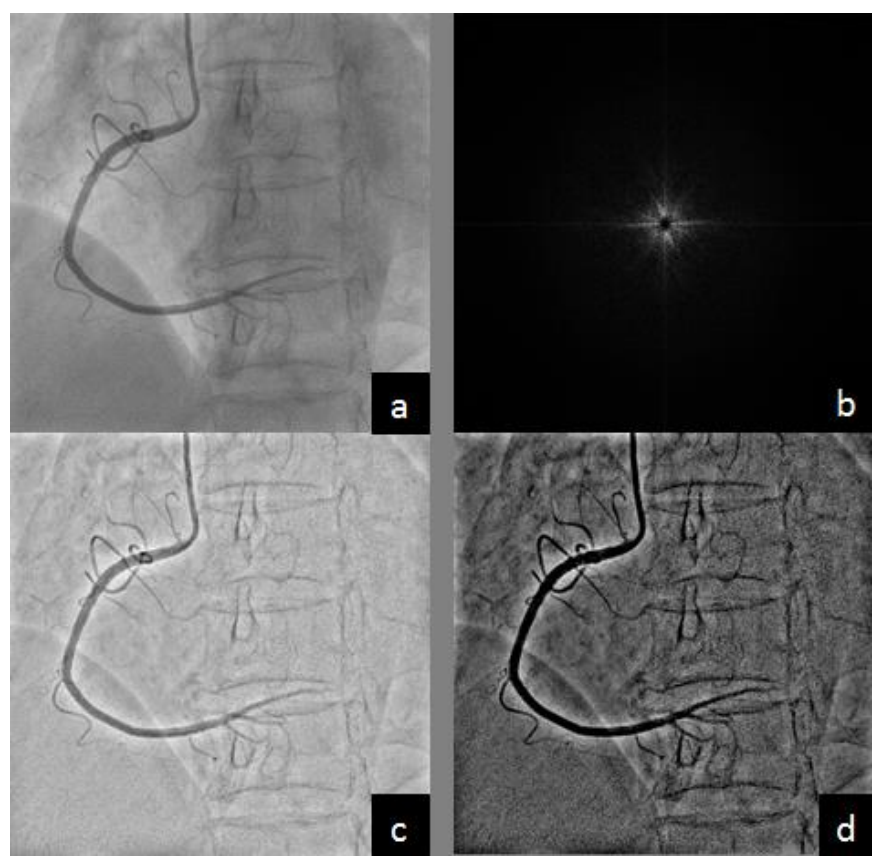

Fig. 2 Proposed frame enhancement method; (a) direct CCA frame; (b) frequency spectrum obtained from DFT; (c) Homomorphic filtered frame; (d) contrast stretched frame.

\section{Calculating Motion Vector}

Motion vectors among the two consecutive frames of the CCA are processed iteratively to correct the epicardial deformations within the frames. Initially, it calculates the motion vectors between the first and the second frames of the CCA to be processed and the motion stabilized frame is produced as the output. From the second iteration onwards, it takes the motion stabilized frame produced in the previous step and the next frame in the input CCA as inputs to calculate the motion vectors. Hence, it produces the subsequent motion stabilized frames as output. Moreover, this phase has been implemented based on the technique called optical flow because it can compute the motion among the two consecutive frames of the CCA to be processed. Additionally, optical flow is $2 \mathrm{D}$ vector field where each vector is a displacement vector showing the movement of points from the first frame to the second [25]. The noise reduced and uniformly illuminated contrast stretched frames are the input to this phase to obtain the matrix of calculated motion vectors as the output.

Initially, the Harris corner detector was applied to the current frame to detect the good features to track in the next frame [26]. Moreover, the corners were detected in sub pixel level to improve the accuracy. In the later stage of this phase, Lucas-Kanade optical flow is applied to iteratively track those points in the next consecutive frame [25]. Image (a) and (b) of Fig. 3 depict two consecutive frames of a selected CCA and image (c) of the same figure depicts the computed motion vectors ( marked using lines) based on the tracked feature points in those two frames.

\section{E. Creating Stabilized Frames}

The objective of this phase is to correct the epicardial deformations in the frame and obtain the stabilized CCA frames. It begins after tracking the corners in current frame which are identical to the corners in the previous frame. Within the phase, it calculates the $3 \times 3$ homography matrix based on the correspondence of marked points in two consecutive frames. In order to obtain the stabilized frame, it is required to change the perspective of the frame using the transformation matrix obtained during the application of homography. Hence, the perspective warping has been applied to the current frame to obtain the stabilized frame by evading epicardial deformations. Image (d) of Fig. 3 clearly depicts the stabilized frame for further clarifications. The proposed algorithm is as follows;

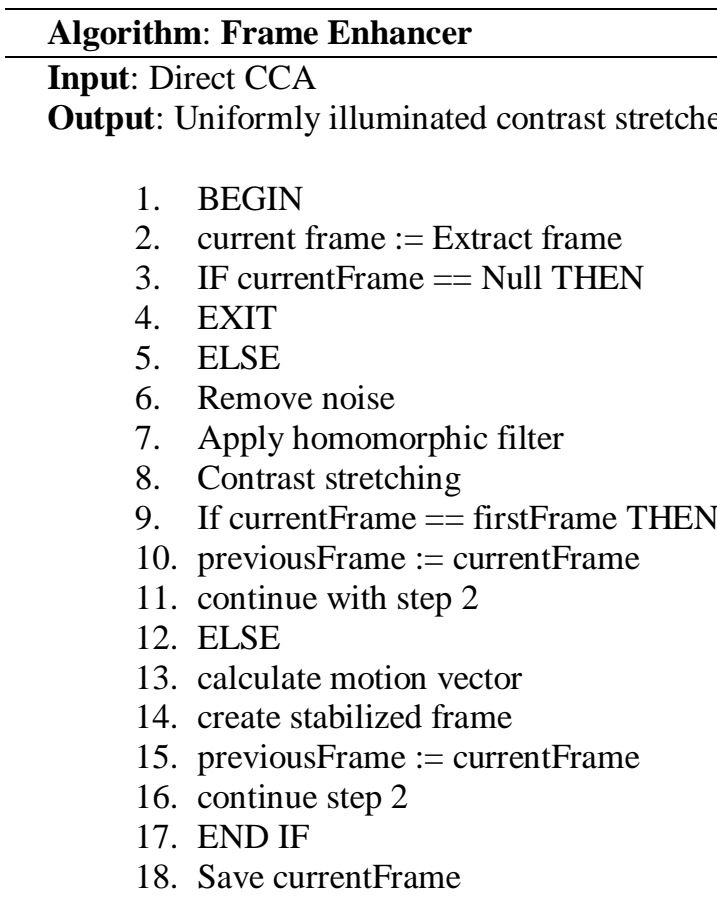


19. END IF

20. END

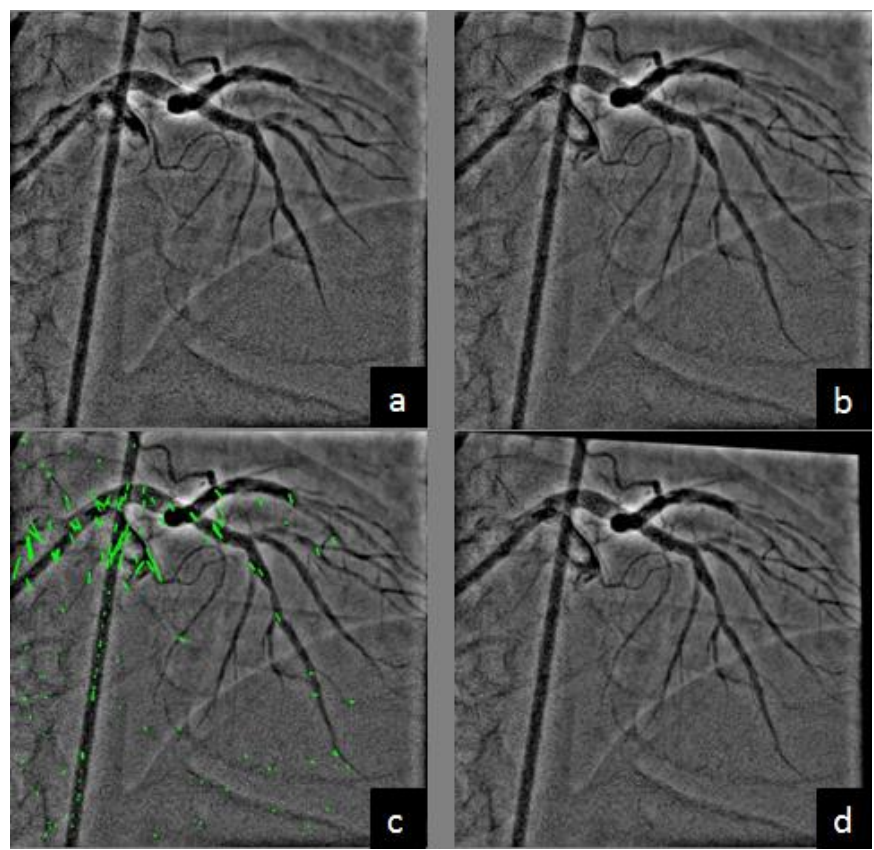

Fig. 3 Steps of proposed method; (a) and(b) two consecutive frames; (c) application of optical flow and computed motion vectors; (d) motion stabilized frame after application of homography and warping.

\section{RESULTS}

This section elaborates the experimental methods and the results of this study in detail. Initially, it explains the method followed to extract the data for validation. Afterwards, the four different experimental methods carried out to validate the proposed method and the results of each of those methods have been presented in the later sub sections.

\section{A. Data Extraction}

The direct CCAs produced by Philips Medical System were used for the experiments and those were recorded in the frame rate of $15 \mathrm{fps}$ with $512 \times 512$ resolution. In order to create a data set to validate the proposed method, thirty direct CCAs were extracted under Left Anterior Oblique Cranial (LAO-CRA) view, Anterior Posterior Caudal (AP-CODL) view and Anterior Posterior Cranial (AP-CRA) view because those views provide excellent visualizations for the main $\mathrm{CA}$ namely RCA, CX and LAD respectively. Further, this dataset consists of 400 total frames. Ethics review committee of the Faculty of Medicine, University of Colombo granted the ethical clearance for extracting these clinical data from the Cardiology unit, National Hospital of Sri Lanka.

\section{B. Qualitative Assessment}

In order to visually compare the enhancement results, 400 direct CCA frames were enhanced using the proposed method. In order to visually compare the results, the direct CCA frames in the data set and the respective enhanced frames were listed sequentially.

Fig. 4 depicts the sample illustrations of the three selected direct CCA frames with the respective enhancement results of them consecutively by covering three main CAs namely, LAD, CX and RCA.

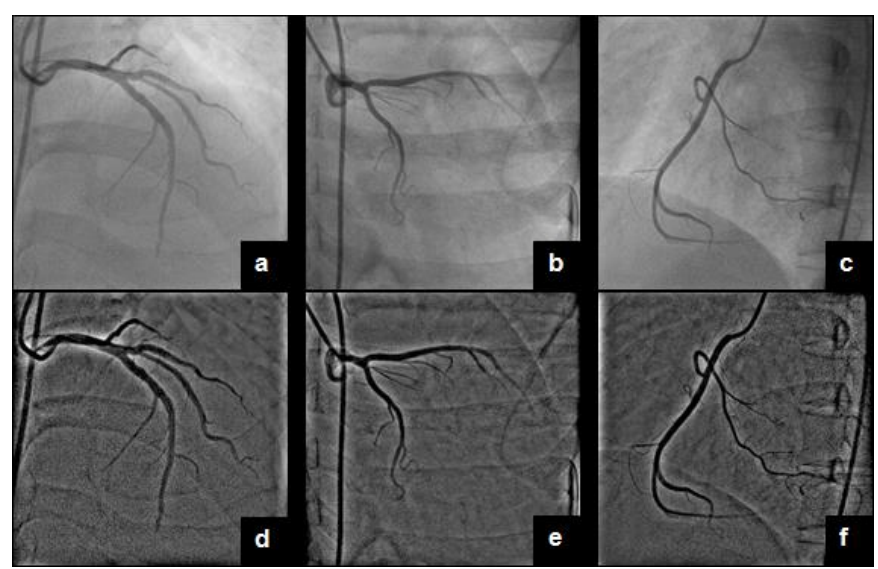

Fig. 4 Frame enhancement results; (a)(b)(c) direct CCA frames representing LAD, CX and RCA; (d)(e)(f) enhanced frames of LAD,CX and RCA

\section{Comparison with Recent Methods}

Moreover, the proposed enhancement method is compared with some of the selected latest enhancement methods proposed in resent past publications which are based on adaptive histogram equalization and CLAHE method (Lara et.al) [17][18]. Visual illustrations of the frames obtained from those published methods and respective intensity histogram are clearly depicted in Fig. 5 to benchmark the proposed enhancement method.

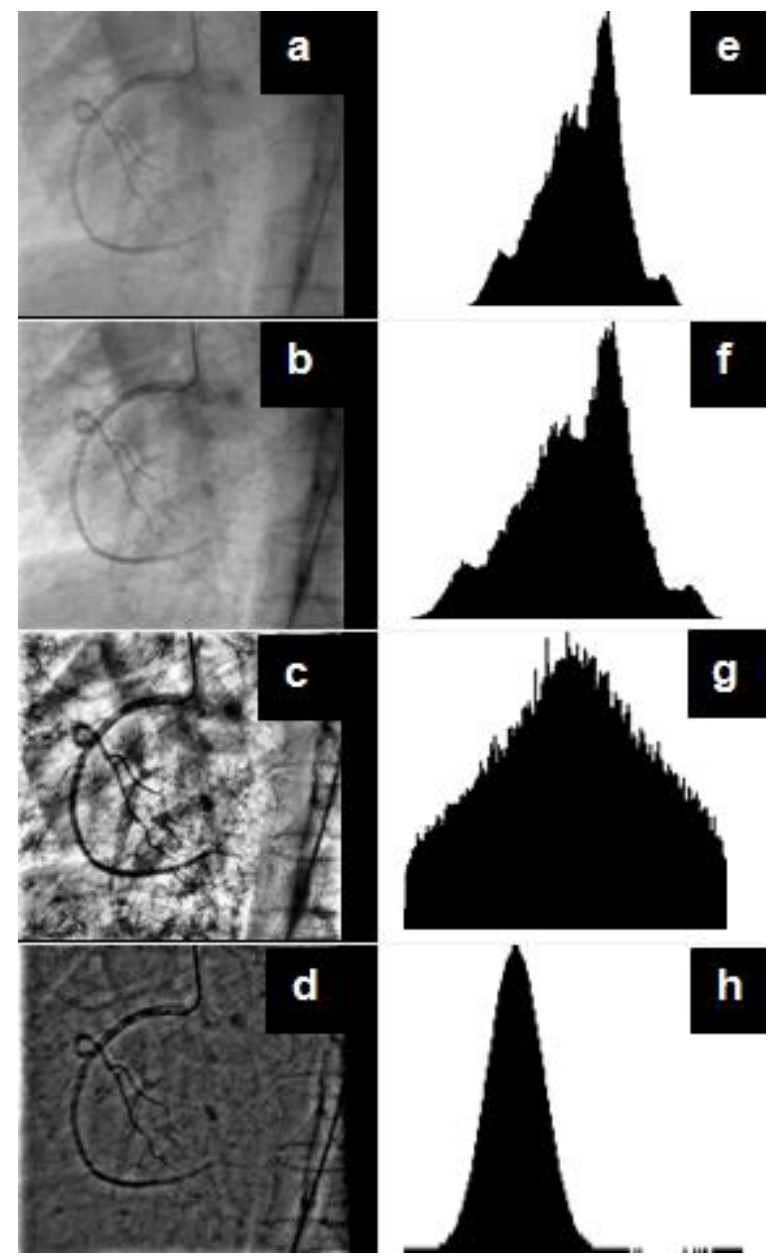

Fig. 5 Comparison of enhancement methods (a) original frame; (b) adaptive histogram equalization; (c) CLAHE; (d) Proposed method; (e),(f),(g) and (h) intensity histograms. 


\section{Quantitative Assessment}

In order to quantitatively evaluate the visual quality among the proposed method and the CLAHE method, the frame entropy based validation method has been proposed.

Entropy (E) of a grayscale image returns a scalar value representing the statistical measure of randomness that can be used to characterize the texture of the input image. It is defined as shown in (7);

$$
E=-\sum_{k} p_{k} \log _{2} p_{k}
$$

where, $k$ is the number of gray levels and $p_{k}$ is the probability associated with gray level $k$.

Within this experiment, 50 randomly selected direct CCA frames covering three main CAs were selected from the data set. Then, the entropy was calculated for each of those selected frames after enhancing them by using CLAHEE method and the proposed method described in this study. Fig. 6 depicts the distribution of the calculated entropy of those frames.

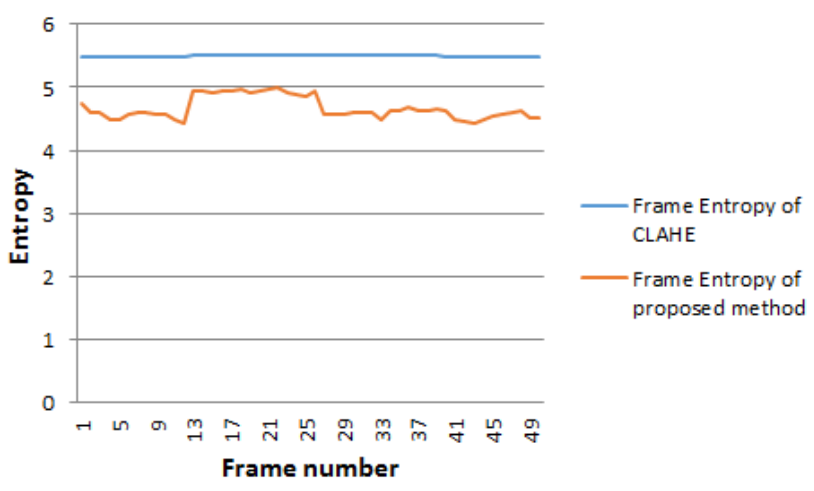

Fig. 6 Entropy distribution of CLAHEE and proposed method.

\section{E. Visual Assessment of Motion Stabilization Method}

In order to qualitatively assess the impact of proposed motion stabilization method, 30 direct CCAs in the data set were processed using the proposed motion stabilization method. In order to visually compare the results, the enhanced CCA frames in the data set and the respective motion stabilized frames were listed sequentially. Fig. 7 depicts the four consecutive frames of a selected enhanced CCA and the identical stabilized frames to visually analyze the robustness of the proposed motion stabilization method.

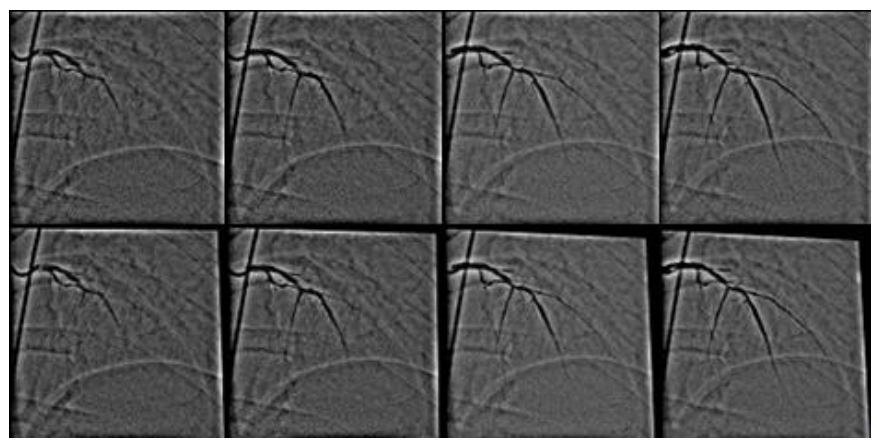

Fig. 7 Correction of epicardial deformations. (Top row depicts four consecutive frames of an enhanced CCA and bottom row depicts the stabilized frames)

\section{DisCUSSION}

The objective of this section is to critically discuss the results of this proposed frame enhancement method. Nonuniform illumination is a definite visual degradation effect recorded in the CCA frames and in this proposed enhancement method, the homomorphic high pass filter has been applied to cut off the frequencies, which are responsible for the illumination. As a result of that, it would be possible to save the frequencies, which are reflected from the desired objects significantly. Hence, this frame enhancement method has provided excellent visualizations of the CCAs. The applied contrast stretching technique also improves the contrast of the frame and it further improves the visualization of CA so that it can be supportive for the diagnosis. Additionally, this homomorphic filter based enhancement does not amplify the noise components in the frames to be processed because it performs the frame enhancement by subtracting unnecessary frequency bands from the original frame. Fig. 4 depicts the sample frame enhancement results for visually analyzing the results. Compared to the direct CCA frames depicted in Fig. 4, the enhanced frames have clearly emphasized the vessel network dark in color. It is clear that most of the background structures such as ribs, soft tissues in heart chambers and pleura were deemphasized to improve the contrast of the vasculature. Further, imbalance illumination patterns shown in original frames were completely eliminated in the resulting frames. Moreover, the capillaries are also clearly emphasized within the enhanced frames.

In this study, setting a value for parameter $D_{0}$ is also done through an experiment work. $D_{0}$ describes the distance from origin to cutoff frequency in the DFT frequency spectrum and is used by the homomorphic filter mentioned in (4). Logically $D_{0}$ can vary from $0-256$ interval. A prior experiment was carried out to determine the best value for this parameter. During the experiment, the intensity histograms of the three CCA frames of three selected angiography views were tested by setting the integer values for $D_{0}$. Fig. 8 depicts the selected results to provide the effect. According to the results, it would be able to visually analyze the contrast of the tested frames which were gradually increased when $D_{0}$ is changing from 1 to 10 and reduced after that. Moreover, the dynamic range of the intensity histogram was also stretched maximally at $D_{0}=10$. These facts are clearly depicted in Fig. 8 and therefore 10 is set as the best value for $D_{0}$ parameter.

Fig. 5 visually illustrates the consequences of various image enhancement methods after applying them to a direct CCA frame. Fig. 5 (a) and (e) depicts the original CCA frame and its intensity histogram whereas Fig. 5(b) and (f) depict the adaptive histogram equalization result of direct CCA frame shown in image (a) and its intensity histogram respectively. Determining the uniform threshold values for implementing the adaptive histogram equalization is the major difficulty of this method. Moreover, the non-uniform threshold values cause failures or loss of spatial connectivity of the vessel structures and it leads to incorrect vessel segmentation when this enhancement frames have been used in quantitative coronary analysis. Lara and team have used CLAHE method as the contrast enhancement technique in 
their proposed angiography segmentation study [17]. Fig. 5(c) and (g) depicts the CLAHE result and the intensity histogram respectively. Even though CLAHE stretches the contrast of the image to obtain the visual quality, it cannot remove illumination frequencies recorded in the angiogram images, which cause the degradation of visual quality in the CCA frames. Moreover, it amplifies the noise signals in the CCA frames. Fig. 5 (d) and (h) depict the enhancement results provided by our proposed method. This image has deemphasized the illumination in the original image and preserved the whole vessel structure as it can be visualized in a clear manner. Moreover, it produced the balance intensity distribution among all the intensity histograms produced by other methods.

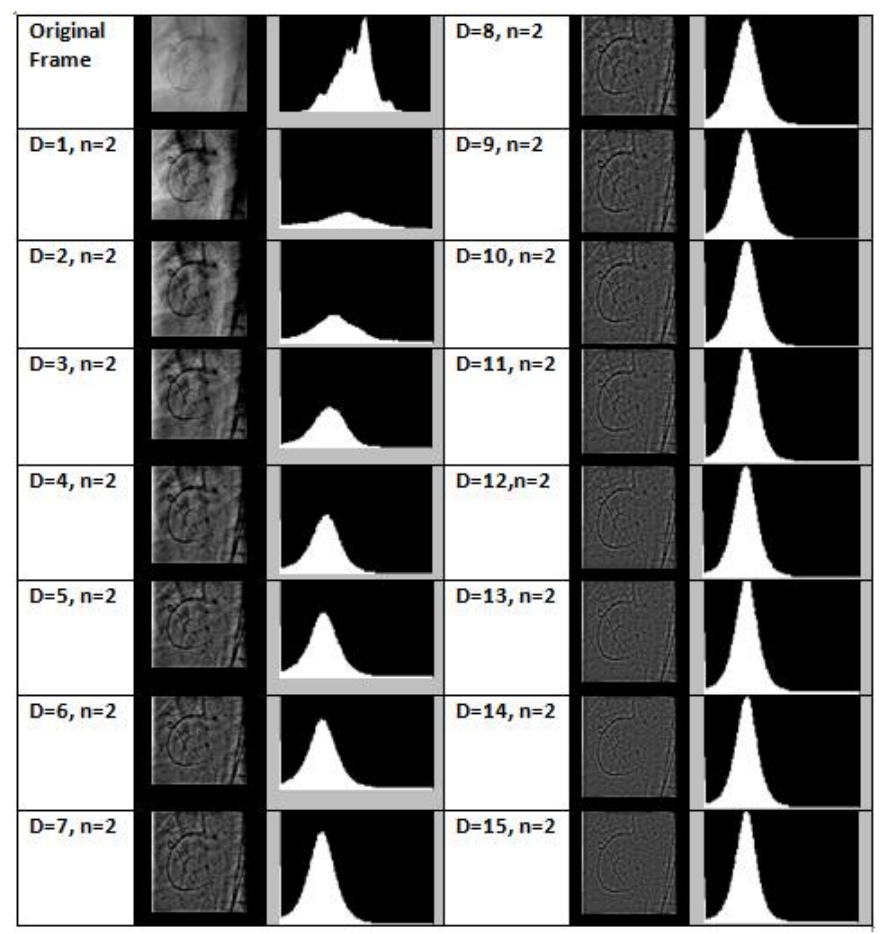

Fig. 8 Parameter analysis for $D_{0}$ in homomorphic filter.

The entropy provides the amount of information in the image. Both the number of unique spatial samples and unique spectral values contribute to the entropy in different weights [27][28]. Any typical contrast enhancement technique would reduce the entropy because some information can vanish due to intensity saturation (at 255 or at 0 ). If the contrast enhancement technique simultaneously reduces the illumination variation also, then the entropy will be extremely small. The reasons are the illumination variation also contributes to the entropy and the number of unique intensities becomes lower as the illumination is being normalized. This feature clearly emphasized in the graph shown in Fig. 6. According to Fig. 6, the calculated frame entropy of the proposed method is lower than the entropy of CLAHE. Hence, this proves how well the illumination is normalized and how well the unnecessary details such as noise were suppressed in the processed frames.

Chen and his team have implemented a CCA enhancement method based on an improved multi-scale Hessian matrix combined with morphological top-hat operation [15]. In order to calculate Hessian matrix, it is required to obtain the derivative images of the angiography images to be processed. These derivative images can be calculated by applying convolution of the second order partial derivatives of a Gaussian filter. Application of Gausian filter leads to loss of image data and it violates image enhancement standards mentioned in the related work section in this section.

Fig. 7 depicts the visual illustrations of the proposed motion stabilization step. According to the figure, during the motion stabilization, it would be able to correct some epicardial dynamics occurred during the angiography procedure. It was calculated during the application of optical flow and corrected during the calculation of homography based affine transformation. As the results of the correction, a set of transformed frames were generated and those frames are free from the epicardial motion.

Research work in [9] has been reported a motion stabilization method in CCAs using the SIFT feature descriptor. According to this study, the global motion vector has been determined based on SIFT feature matching between the two consecutive frames. It has been empirically observed that SIFT provides many miss-corresponding key point pairs in angiography and feature matching as depicted in Fig 9. This illustration clearly emphasizes that most of the detected feature points are located in the background of the frame. Moreover, the detected feature points in vessel structures have not performed correct feature matching with the corresponding frame for all detected feature points. As a result of that, it provides erroneous motion vectors for motion compensation.

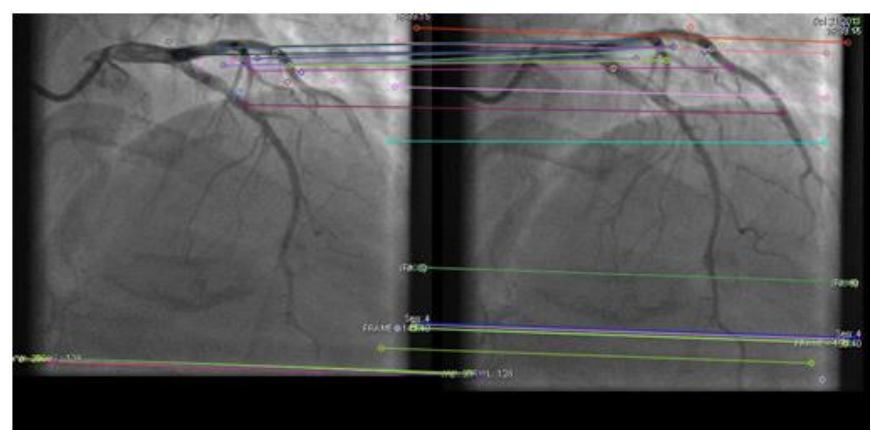

Fig. 9 SIFT feature matching results.

By analyzing aforementioned factors, it can be concluded that this proposed frame enhancement method provides a better visualization of the CA vasculature without any loss in the quality of the image. Further, the original dimensions of the vascular structures are also being preserved within this enhancement technique and it is a mandatory requirement to implement a quantitative coronary analysis based on angiography.

As the future experiments, the enhanced CCA produced in this study can be tested in cath lab for real time evaluations. Further, the algorithms developed in this study can be used to do some experiments on developing angiography based quantitative coronary analysis techniques. Therefore, it can be concluded that the results provided by this study lay the foundation for implementing a mechanism to automatic segmentation of CA vasculature to objectively assess the severity of the Coronary Artery Disease and it could be the next extension of this research. 


\section{CONCLUSION}

In this study, we proposed a frequency filtering based frame enhancement method for CCA to improve its diagnostic visualization. The proposed method is based on homomorphic butterworth high pass filter and empirically validated contrast stretching technique. In order to correct the epicardial deformations, an optical flow based frame stabilization method has been applied in the later stage of the proposed method. The empirical results have proven that it provides a better contrast to visualize the CA vasculature rather than the widely accepted conventional approaches like CLAHE and an adaptive threshold. Further, the study results have clearly emphasized the visual improvement obtained through this novel method.

According to the results, it is clear that this enhancement method can be recommended for the application on clinical procedures because it visualizes the $\mathrm{CA}$ vasculature with high contrast. Further, this proposed method does not make any negative impact to disappear the detailed image structures during the enhancing procedure. Lastly, it has been revealed that the original dimensions of vascular structures should be preserved in the enhanced image. Hence, it can be concluded that this proposed angiography enhancement method preserved the reported standards in the area of angiographic image enhancement, which have been widely accepted by cardiologists [13]. As the future experiments, the enhanced CCA produced in this study can be used to do some experiments on developing angiography based quantitative coronary analysis techniques.

\section{ACKNOWLEDGMENT}

The authors thank the staff of the Cardiology Unit of the National Hospital Sri Lanka.

\section{REFERENCES}

[1] Kulathilake K.A.S.H., Ranthunga L., Constantine G. \& Abdulla N.A. (2017). Visual alignment of arteries in coronary cine-angiogram using global motion stabilisation approach. J. Natl. Sci. Found. Sri Lanka, 45(1), pp-41-51.

[2] Fhea A. W. Bs. Ms. C. S. R. \& Rgn A. G. Bs. P.(2006). Ross and Wilson Anatomy and Physiology in Health and Illness, 10th ed. Churchill Livingstone.

[3] Shaw A, Smith B. \& Howlett D.C. (2011). Axial CTA of the heart in FRCR Part 1 Anatomy Mock Examinations, p.-61, Cambridge University Press.

[4] Boron W.F. \& Boulpaep E.L. (2012). The heart in Medical Physiology, 2e Updated Edition: with Sudent Consult Online Access, p.- 581, Elsevier Health Sciences

[5] Takagi A., Tsurumi Y., Magosaki N., Suzuki K., Nakamura K. \& Kasanuki H. (1999). Significance of angiographic haziness at the distal stent edge: analysis by intravascular ultrasound and quantitative coronary angiography. J. Cardiol., 33(6), pp-307-316.

[6] Dehkordi M.T., Sadri S. \& Doosthoseini A. (2011). A review of coronary vessel segmentation algorithms. J. Med. Signals Sens., 1(1), pp- 49-54.

[7] Wang W., Li C., Cai W. \& Wu X. (2010). Coronary angiography image edge detection based on information measure.WASE International Conference on Information Engineering (ICIE), 1, pp$141-144$.

[8] Khan M.A.U, Bahadur Khan R.,Bilal R., Jamil A. \& Ali Shah M. (2008). Enhancement of angiogram images using pseudo color processing. Inf. Technol. J., 7(1), pp-210-214.

[9] Kusumawardhani A., Mengko T., Fahri I., Soerianata S., Firman D. \& Zakaria H. (2011). Coronary angiogram stabilization for OuBE values calculation using SIFT method. 2nd International Conference on Instrumentation, Communications, Information Technology, and Biomedical Engineering, pp-111-116.
[10] Haris K., Efstratiadis S.N., Maglaveras N., Pappas C., Gourassas J. \& Louridas G. (1999). Model-based morphological segmentation and labeling of coronary angiograms. IEEE Trans. Med. Imaging, 18(10), pp-1003-1015.

[11] Schrijver M. (2002). Angiographic image analysis to assess the severity of coronary stenosis. Doctoral Thesis, Universiteit Twente, Enschede.

[12] Anne W. \& Allison G. (2010). Ross and Wilson Anatomy and Physiology in Health and Illness, 11th ed. Edinburgh; New York: Churchill Livingstone.

[13] Tu S. (2009). Coronary angiography enhancement for visualization. Int. J. Cardiovasc. Imaging, 25(7), pp-657-667.

[14] Khan M.A.U., Khan M.K. \& Khan M.A. (2004). Coronary angiogram image enhancement using decimation-free directional filter banks. IEEE International Conference on Acoustics, Speech, and Signal Processing Proceedings, 5, pp-441-444.

[15] Chen K., Yin Q., Jia X. \& Lu M. (2015). Image enhancement based improved multi-scale hessian matrix for coronary angiography. Int. J. Comput. Appl., 126(10), pp-1-4.

[16] Tu S. (2009). Coronary angiography enhancement for visualization. Int. J. Cardiovasc. Imaging, 25(7), pp-657-667.

[17] Lara D.S.D, Faria A.W.C., de A. Araújo A. \& Menotti D. (2013). A novel hybrid method for the segmentation of the coronary artery tree in $2 D$ angiograms. Int. J. Comput. Sci. Inf. Technol., 5(3), pp-45-65.

[18] Hanan, Ahmed S.S. \& Nordin J. (2011). Improving diagnostic viewing of medical images using enhancement algorithms. J. Comput. Sci., 7(12), pp-1831-1838.

[19] Pahlm O. \& Wagner G.S. (2011). Multimodal Cardiovascular Imaging: Principles and Clinical Applications, McGraw Hill Professional.

[20] Lee T.F, Lee C.Y., Chao P.J., Lee C., Wang C.Y. \& Fang C.H. (2009) Quantitative coronary analysis medical image processing improved by combining wavelet edge detection and segmentation. Fourth International Conference on Innovative Computing, Information and Control, pp-1196-1199.

[21] Kumar M.S.D., Shen D., Wei L., Turlapthi R. \& Suri J.S. (2007). Motion correction strategies for interventional angiography images: a comparative approach. IEEE International Conference on Image Processing, 1, pp- $497-500$.

[22] Meunier J., Bourassa M.J., Bertrand M., Verreault M. \& Mailloux G.E. (1989). Regional epicardial dynamics computed from coronary cineangiograms. Computers in Cardiology Proceedings, pp-307-310.

[23] Wang Y., Riederer S.J. \& Ehman R.L. (1995). Respiratory motion of the heart: Kinematics and the implications for the spatial resolution in coronary imaging. Magn. Reson. Med., 33(5), pp-713-719.

[24] Zheng S. \& Weirong D. (2009). Analysis of coronary arterial dynamics from $x$-ray angiographic sequences. Second International Symposium on Computational Intelligence and Design, 1, pp-201 204.

[25] Lucas B.D. \& Kanade T. (1981). An iterative image registration technique with an application to stereo vision. Imaging Underst. Workshop, pp-121-130.

[26] Harris C. \&Stephens M. (1988). A combined corner and edge detector. In Proc. of Fourth Alvey Vision Conference, pp-147-151.

[27] Tsai D.Y., Lee Y. \& Matsuyama E. (2008). Information entropy measure for evaluation of image quality. J. Digit. Imaging, 21(3), pp-338-347.

[28] Celik T. (2014). Spatial entropy-based global and local image contrast enhancement. IEEE Trans. Image Process., 23(12), pp-52985308. 